\title{
A facile synthesis of flavones using recyclable ionic liquid under microwave irradiation
}

\author{
Swapnil R. Sarda, Mohsin Y. Pathan, Vijaykumar V. Paike, Pandurang R. Pachmase, \\ Wamanrao N. Jadhav, and Rajendra P. Pawar* \\ Organic Chemistry Synthesis Laboratory, Dnyanopasak College, Parbhani-431401, India \\ E-mail:rppawar@yahoo.com
}

\begin{abstract}
A high yielding and fast method for the smooth conversion of substituted 1-(2-hydroxyphenyl)3-phenyl-1,3-propane diones to the corresponding 2-phenyl-4H-chromen-4-ones under microwave irradiations using ionic liquid $\left[\mathrm{EtNH}_{3}\right] \mathrm{NO}_{3}$ is reported.
\end{abstract}

Keywords: Flavones, ionic liquid, microwave irradiation, 1,3-dicarbonyl compounds, dehydrative cyclization

\section{Introduction}

Flavones are important naturally occurring organic compounds possessing wide range of biological activities ${ }^{1}$ used in the treatment of various diseases. ${ }^{2}$ Different methods are used for the synthesis of flavones, includes Allan-Robinson synthesis, ${ }^{3}$ chalcones ${ }^{4}$ and Wittig reaction. ${ }^{5}$ The most common method used involves Baker-Venkatraman rearrangement. In this method 2hydroxyacetophenone was converted to its benzoyl ester, which further in presence of base (pyridine/KOH) gives the 1,3-diketone. The diketones are further cyclized under strongly acidic conditions to afford the corresponding flavones. ${ }^{6}$ In recent developments of such dehydrative cyclization various methods have been used, i.e., the use of Amberlyst $15,{ }^{7} \mathrm{Co}^{\mathrm{III}}$ (sulfur) $\mathrm{OH},{ }^{8}$ $\mathrm{FeCl}_{3},{ }^{9} \mathrm{Br}_{2} / \mathrm{CHCl}_{3},{ }^{10} \mathrm{EtOH} / \mathrm{HCl},{ }^{11}$ clay, ${ }^{12} \mathrm{NaOAc} / \mathrm{AcOH},{ }^{13}$ and $\mathrm{H}_{2} \mathrm{SO}_{4}$ under microwave irradiation. $^{14}$

The chemical and pharmaceutical industries are always under pressure to develop more environmentally friendly organic reaction methodologies. Therefore, microwave irradiation is used for a variety of organic reactions due to its use in more rapid and cleaner syntheses of organic compounds. ${ }^{15-17}$ A high yield synthesis of flavones and chromones has been also reported from 1,3-propanediones in ethanol, using $\mathrm{CuCl}_{2}$ catalyst under microwave irradiation. ${ }^{18}$ Recently, significant progress has been made in the application of ionic liquids in catalytic processes. $^{18,19}$ Ionic liquids are the salts of organic heterocyclic cations and inorganic anions. 
They exist in liquid state at ambient temperature; hence the reactions in presence of ionic liquids need no additional solvent. Ionic liquids have attracted much attention due to their mild reaction conditions, short reaction times and better yield, solvating ability, and easy recyclability. ${ }^{20}$ Various reactions have been reported recently using ionic liquids as a catalyst, reaction media, ${ }^{21}$ as rate enhancers ${ }^{22}$ and in peptide synthesis. ${ }^{23}$
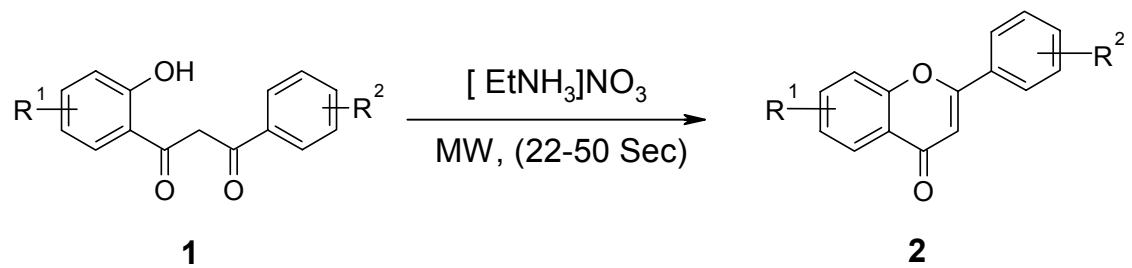

\section{Scheme 1}

\section{Results and Discussion}

Herein we report the synthesis of flavones $\mathbf{2}$ promoted by the ionic liquid catalyst, ethyl ammonium nitrate $\left[\mathrm{EtNH}_{3}\right] \mathrm{NO}_{3}$ under microwave irradiation, in excellent yield with shorter reaction time (Scheme 1). The ionic liquid can be recycled and reused several times. The ionic liquid $\left[\mathrm{EtNH}_{3}\right] \mathrm{NO}_{3}$ was prepared by the literature method. ${ }^{24}$

In a typical reaction, the 1,3-diketones $\mathbf{1}$ in ionic liquid $\left[\mathrm{EtNH}_{3}\right] \mathrm{NO}_{3}$ was irradiated in a domestic microwave oven for a specified time period. The progress of reaction was monitored by TLC. After completion of the reaction, extraction with organic solvent followed by aqueous work-up afforded pure flavones 2 in $80-90 \%$ yield. The ionic liquid is water-soluble and therefore goes into the aqueous layer. The aqueous layer was distilled at $80{ }^{0} \mathrm{C}$ under vacuum to remove water, leaving behind the ionic liquid (about 90\%), which was recycled for several times to carry out the same experiment.

To evaluate the synthetic utility of the process, various substituted diketones were prepared by the established procedure ${ }^{6}$ and subjected to the reaction under microwave irradiation (Table 1). The reaction proceeds cleanly without formation of any side product except water. The protocol of the process offers advantages in terms of simple procedure and work up, mild reaction conditions and excellent yields. The ionic liquid used for reaction was recovered and reused with identical results. Thus, the recyclability was confirmed (Table 2).

The reactions were also carried out in presence of 1,3-dibutylimidazolium bromide [bbim]Br ionic liquid for comparison. The resulting flavones are formed with similar yield. 


\section{Conclusions}

In summary, we demonstrated an efficient and mild protocol for the dehydrative cyclization of 1,3-diaryl diketones to flavones in the presence of the ionic liquid $\left[\mathrm{EtNH}_{3}\right] \mathrm{NO}_{3}$ under microwave irradiation. Shorter reaction time, simple reaction conditions, and higher yield render this microwave irradiation method superior. The method is clean and simple, which may be used as an alternative to the existing methods.

\section{Experimental Section}

General Procedures. Melting points were determined in open glass capillaries and are uncorrected. ${ }^{1} \mathrm{H}$ NMR and ${ }^{13} \mathrm{C}$ NMR spectra were recorded at room temperature on a $300 \mathrm{MHz}$. Varian Inova Spectrometer in $\mathrm{CDCl}_{3}$ using TMS as internal standard. A Samsung domestic microwave oven was used at $80^{\circ} \mathrm{C}(150 \mathrm{~W}$ power $)$ for all the experiments.

General procedure for the preparation of 2-phenyl-4H-chromen-4-one (2a-h). The 1,3diaryl diketone $1(1 \mathrm{mmol})$ was added in ionic liquid $\left[\mathrm{EtNH}_{3}\right] \mathrm{NO}_{3}(2 \mathrm{mmol})$ and irradiated in a microwave oven for 22-50 seconds. The reaction was monitored by TLC. After completion of the reaction, the mixture was extracted with $(3 \times 20 \mathrm{ml})$ ethyl acetate: petroleum ether $(1: 1)$. The organic layer was washed with water and dried over anhydrous $\mathrm{MgSO}_{4}$. Organic solvent was evaporated and solid was crystallized from petroleum ether to afford pure flavones $\mathbf{2}$. Further, the aqueous layer was distilled at $80{ }^{\circ} \mathrm{C}$ under vacuum to remove water, leaving behind the ionic liquid (about 90\%), which was recycled several times to carry out the same experiment (Table 2). The products $\mathbf{2 a}-\mathbf{h}$ were confirmed by comparison with authentic samples, ${ }^{1} \mathrm{H}$ NMR, and melting points.

2-(4-Chlorophenyl)-4H-chromen-4-one (2b). ${ }^{1} \mathrm{H}$ NMR (300 MHz, $\left.\mathrm{CDCl}_{3}\right) \delta 2.44$ (s, 3H), 7.22 (s, 1H), $7.69(\mathrm{dd}, 1 \mathrm{H}, \mathrm{J}=8.9,2.1 \mathrm{~Hz}), 7.74(\mathrm{~d}, 1 \mathrm{H}, \mathrm{J}=8.6 \mathrm{~Hz}), 7.85(\mathrm{~m}, 1 \mathrm{H}), 8.38(\mathrm{~s}, 4 \mathrm{H}) ;{ }^{13} \mathrm{C}$ NMR $\left(300 \mathrm{MHz}, \mathrm{CDCl}_{3}\right)$ 21.5, 109.9, 119.6, 125.1, 125.3, 128.8, 136.5, 136.8, 138.3, 149.7, 154.8, 160.6, 177.2.

2-(2,4-dimethoxyphenyl)-4H-chromen-4-one (2e). ${ }^{1} \mathrm{H}$ NMR (300 MHz, $\left.\mathrm{CDCl}_{3}\right) \delta 3.95$ (s, 3H), $3.98(\mathrm{~s}, 3 \mathrm{H}), 6.72(\mathrm{~s}, 1 \mathrm{H}), 6.96(\mathrm{~d}, 1 \mathrm{H}, \mathrm{J}=8.1 \mathrm{~Hz}), 7.36(\mathrm{~d}, 1 \mathrm{H}, \mathrm{J}=1.8 \mathrm{~Hz}), 7.40$ (dd, 1H, J = 7.2 $\mathrm{Hz}, \mathrm{J}=7.5 \mathrm{~Hz}$ ), 7.51 (dd, 1H, J = 1.8 Hz, J = 8.1 Hz), 7.53 (d, 1H, J = 7.5 Hz), 7.67 (ddd, 1H, J $=1.5,7.2,7.8 \mathrm{~Hz}) 8.20(\mathrm{dd}, 1 \mathrm{H}, \mathrm{J}=1.5,7.8 \mathrm{~Hz}) ;{ }^{13} \mathrm{C} \mathrm{NMR}\left(300 \mathrm{MHz}, \mathrm{CDCl}_{3}\right) 56.1,106.4$, 108.8, 111.2, 118.0, 120.0, 123.9, 124.2, 125.6, 133.6, 149.3, 152.1, 156.1, 163.3, 178.3.

7-Hydroxy-2-phenyl-4H-chromen-4-one (2f). ${ }^{1} \mathrm{H}$ NMR $\left(300 \mathrm{MHz}, \mathrm{CDCl}_{3}\right) \delta 6.91(\mathrm{~s}, 1 \mathrm{H}), 7.95$ $(\mathrm{dd}, 1 \mathrm{H}, \mathrm{J}=1.8, \mathrm{~J}=9.0 \mathrm{~Hz}), 7.02(\mathrm{~d}, 1 \mathrm{H}, \mathrm{J}=1.8 \mathrm{~Hz}), 7.57-7.29(\mathrm{~m}, 3 \mathrm{H}), 7.91(\mathrm{~d}, 4 \mathrm{H}, \mathrm{J}=9.0$ $\mathrm{Hz}), 8.05-8.08(\mathrm{~m}, 2 \mathrm{H}) ; 10.8(\mathrm{~s}, 1 \mathrm{H}) ;{ }^{13} \mathrm{C} \mathrm{NMR}\left(300 \mathrm{MHz}, \mathrm{CDCl}_{3}\right)$ 102.6, 106.6, 115.1, 116.2, 126.2, 126.5, 129.1, 131.3, 131.5, 157.5, 161.2, 162.8, 176.4 . 
Table 1. Synthesis of flavones (2a-h) using ionic liquid (EAN) under microwave irradiation

a<smiles>O=C(CC(=O)c1ccccc1O)c1ccccc1</smiles>

b<smiles>O=C(CC(=O)c1ccccc1O)c1ccc(Cl)cc1</smiles>

c<smiles>COc1ccc(C(=O)CC(=O)c2ccccc2O)cc1</smiles>

d<smiles>Cc1ccc(O)c(C(=O)CC(=O)c2ccc([N+](=O)[O-])cc2)c1</smiles>

e<smiles>COc1ccc(C(=O)CC(=O)c2ccccc2O)cc1OC</smiles>

f<smiles>O=C(CC(=O)c1ccc(O)cc1O)c1ccccc1</smiles><smiles>O=C(CC(=O)c1ccccc1Cl)c1ccccc1O</smiles>

g

$\mathrm{h}$<smiles>COc1cccc(C(=O)CC(=O)c2ccccc2O)c1</smiles><smiles>O=c1cc(-c2ccccc2)oc2ccccc12</smiles>

50

81

97

45

90

185-187

250

83

155-156

40

88

277<smiles>C/C=C/c1cc(=O)c2cc(C)ccc2o1</smiles>

22

89 154

35

80 240-241

40

87

118

45

90

128-129

\footnotetext{
a Isolated yield

${ }^{\mathrm{b}}$ Melting points are uncorrected and compared with reported compounds reference 14.
} 
Table 2. Ionic liquid EAN recovered in flavones $\mathbf{2 a}-\mathbf{h}$ synthesis

\begin{tabular}{ccccccc}
\hline & & & \multicolumn{3}{c}{ Yield (\%) } \\
\cline { 5 - 7 } Entry & $1,3-$-Diketone & Product & Time (sec.) & Cycle 1 & Recycle 1 & Recycle 2 \\
\hline 1. & $1 \mathrm{a}$ & $2 \mathrm{a}$ & 50 & 93 & 91 & 91 \\
2. & $1 \mathrm{~b}$ & $2 \mathrm{~b}$ & 45 & 90 & 88 & 88 \\
\hline
\end{tabular}

\section{Acknowledgements}

The authors are thankful to the Principal P. L. More, Dnyanopasak College, Parbhani, for encouragement during the process of carrying out this work.

\section{References}

1. Welton, A. F.; Tobias, L. D.; Fiedler-Nagy, C.; Anderson, W.; Hope, W.; Middleton, K.; Harbirne, J. B.; A. R. Liss, New York, 1986, 231.

2. Havsteen, Biochem. Pharmacol. 1983, 32, 1141.

3. Banerji, A.; Goomer, N. Synthesis 1980, 874.

4. Hoshino, Y.; Oohinata, T.; Takeno, N. Bull. Chem. Soc. Jpn. 1986, 59, 2351.

5. LeFloch'h, Y.; LeFeuvre, M. Tetrahedron Lett. 1986, 27, 2751.

6. (a) Balogh, M.; Laszlo, P. Organic Chemistry using Clays, Springer: Berlin, 1993. (b) Chisen, J.; Chisen, I. C.; Rafelt, J. S.; Macquarrie, D. J.; Clark, J. H. Chem. Commun. 1997, 2203.

7. Hoshino, Y.; Takino, N. Bull. Chem. Soc. Jpn. 1987, 60, 1919.

8. Nishinaga, A.; Ando, H.; Maruyama, K.; Mashino, T. Synthesis 1982, 839.

9. Zubaidha, P. K.; Hashmi, A. M.; Bhosale, R. S. Heterocyclic Commun. 2005, 11, 9100.

10. Garg, S.; Ishar, M. P. S.; Sarin, R.; Gandhi, R. P. Indian J. Chem. Soc. 1994, 33B, 1123.

11. Jung, J. C.; Min, J. P.; Park, O. S. Synth. Commun. 2001, 31, 1837.

12. Verma, R. S.; Saini, R. K.; Kumar, D. J. Chem. Res. (S), 1998, 348.

13. Kumar, P. E.; Prashad, K. J. R. Indian J. Chem. 1999, 38B, 1277.

14. Tsukayama, M.; Kawamura, Y.; Ishizuka, T.; Hayas, S.; Torii, F. Heterocycles, 2003, 60, 2775.

15. (a) Varma, R. S.; Varma, M.; Chatterjee, A. K. J. Chem. Soc., Perkin Trans. 1 1993, 999. (b) Varma, R. S.; Chatterjee, A. K.; Varma, M. Tetrahedron Lett. 1993, 34, 3207. (c) Varma, R. S.; Lamture, J. B.; Varma, M. Tetrahedron Lett. 1993, 34, 3029. (d) Varma, R. S.; Chatterjee, A. K.; Varma, M. Tetrahedron Lett. 1993, 34, 4603. 
16. (a) Lerestif, J. M.; Toupet, L.; Sinbandhit, S.; Tonnerd, F.; Bazureaau, J. P.; Hanelin, J. Tetrahedron, 1997, 53, 6351. (b) Marrero-Terrero, A. L.; Loupy, A. Synlett 1996, 245. (c) Benalloum, A.; Labiad, B.; Villemin, D. Chem. Commun. 1998, 386.

17. (a) El Sayed, H. El Ashry; Kassem, A. A. Arkivoc 2006, (ix), 1. (b) Varma, R. S. J. Heterocycl. Chem. 1999, 36, 1565.

18. (a) Kabalka, G. W.; Mereddy A. R. Tetrahedron Lett. 2005, 46, 6315. Kabalka, G. W.; Mereddy A. R. Tetrahedron Lett. 2005, 46, 6315.

19. Zhao, D.; Wu, M.; Kou, Y.; Min, E. Catal. Today 2002, 2654, 1.

20. (a) Welton, T. Chem. Rev. 1999, 99, 2071. (b) Wassercheid, P.; Keim, W. Angew. Chem. Int. Ed. 2000, 39, 3772. (b) Sheldon, R. Chem. Commun. 2001, 2399. (c) Zhao, D.; Wu, M.; Kou, Y.; Min, K. Catal. Today 2002, 1, 2654.

21. (a) Rajgopal, R.; Jarikote, D. V.; Lahoti, R. J.; Thomas, D.; Srinivasan, K. V. Tetrahedron Lett. 2003, 44, 1615. (b) Jarikote, D. V.; Siddiqui, S. A.; Rajgopal, R.; Thomas, D.; Lahoti, R. J.; Srinivasan, K. V. Tetrahedron Lett. 2003, 44, 1835. (c) Gholap, A. R.; Venkatesan, K.; Thomas, D.; Lahoti, R. J.; Srinivasan, K. V. Green Chem. 2004, 6, 147. (d) Panchgalle, S. P.; Kalkote, U. R.; Nipahadkar, P. S.; Joshi, P. N.; Chavan, S. P.; Chaphekar, G. M. Green Chem. 2004, 6, 308.

22. Madje, B. R.; Shindalkar, S. S.; Shingare, M. S. Indian J. Heterocyclic Chem. 2004, 14, 87.

23. Vallette, H.; Ferron, L.; Coquerel, G.; Guillen, F.; Plaquenent, J. C. Arkivoc 2006, (iv), 200.

24. (a) Walton, T. Chem. Rev. 1999, 99, 2071; Walden, P. Bull. Acad. Imper. Sci. (St. Petersburg), 1914, 1800. (b) Sugden, S.; Wilkins, H. J. Chem. Soc. 1929, 1291. 